\title{
Is Microsatellite Instability Really a Good Prognostic Factor of Colorectal Cancer?
}

\author{
Ui Sup Shin*, Sang Sik Cho*, Sun Mi Moon, Sun Hoo Park ${ }^{1}$, Sun Hee Jee, Eun-Joo Jung², \\ Dae-Yong Hwang ${ }^{2}$ \\ Departments of Surgery and ${ }^{1}$ Pathology, Korea Cancer Center Hospital, Korea Institute of Radiological \& Medical Sciences, Seoul; \\ ${ }^{2}$ Department of Surgery, Colorectal Cancer Center, Kunkuk University Medical Center, Seoul, Korea
}

Purpose: The aim of this study was to investigate the clinicopathologic features of and the prognosis for colorectal cancers (CRCs) with microsatellite instabilities (MSIs).

Methods: Between 2006 and 2009, genotyping was performed on 245 patients with stage II/III CRCs to establish the MSI status. The clinicopathologic differences and the prognostic value of MSI were analyzed. The median follow-up period was 38 months (range, 7-68 months).

Results: Of the total 245 patients, 20 (8.2\%) had MSI-high (H) and 225 (91.8\%) had MSI-low (L) or stable (S) CRCs. Adjuvant chemotherapies were performed on 101 stage II (87.8\%) and 107 stage III patients (82.3\%). Patients with MSI-H CRCs more frequently had a family history of colon cancer $(10 \%$ vs. $2.7 \%, \mathrm{P}=0.003)$, more frequently had a cancer located at the proximal colon $(90.0 \%$ vs. $19.1 \%, \mathrm{P}<0.0001)$, and more often showed a mucinous phenotype or poor differentiation $(35.0 \%$ vs. $7.1 \%, \mathrm{P}=0.001)$. Despite less frequent lymph node metastasis $(25 \%$ vs. $55.6 \%, \mathrm{P}=0.01)$, the number of retrieved lymph nodes was higher $(26.3 \pm 13.1$ vs. $20.7 \pm 1.2, \mathrm{P}=0.04)$ in the MSI-H group. The overall survival and the disease-free survival (DFS) did not differ with respect to MSI status. However, in the stage II subgroup, the DFS for patients with MSI-H CRCs was significantly worse $(72.2 \%$ vs. $90.7 \%, \mathrm{P}=0.03)$. The multivariate analysis performed on this subgroup revealed that MSI$\mathrm{H}$ was an independent poor prognostic factor (adjusted hazard ratio, 4.0; 95\% confidence interval, 1.0-15.6, $\mathrm{P}=0.046$ ).

Conclusion: MSI-H CRCs had distinct clinicopathologic features, and MSI-H was an independent poor prognostic factor in stage II CRCs. Considering the majority of stage II patients were administrated adjuvant chemotherapy, the efficacy of adjuvant chemotherapy for treating MSI CRCs might be different from that for treating MSI-L/S tumors.

\section{Keywords: Microsatellite instability; Colorectal neoplasms; Prognosis; Chemotherapy}

\section{INTRODUCTION}

Colorectal cancer (CRC) is one of the leading causes of death in Western countries. However, in Korea, according to 2011 cancer

Received: August 21, 2013 - Accepted: October 24, 2013

Correspondence to: Sun Mi Moon, M.D.

Department of Surgery, Korea Cancer Center Hospital, Korea Institute of

Radiological \& Medical Sciences, 75 Nowon-ro, Nowon-gu, Seoul 139-706, Korea

Tel: +82-2-970-1237, Fax: +82-2-970-2419

E-mail:msm386@gmail.com

*Ui Sup Shin and Sang Sik Cho contributed equally to this study.

(c) 2014 The Korean Society of Coloproctology

This is an open-access article distributed under the terms of the Creative Commons Attribution NonCommercial License (http://creativecommons.org/licenses/by-nc/3.0) which permits unrestricted noncommercial use, distribution, and reproduction in any medium, provided the original work is properly cited. statistics, CRC is the second most common type of cancer in men, and its incidence is rapidly increasing [1]. Traditional pathological staging systems have been useful in predicting the outcome of CRC, but it is now evident that these cancers are actually heterogeneous. The natural history of CRC is strongly linked to genetic alterations that occur during its progression from an adenoma to a carcinoma and from a carcinoma to metastatic disease [2]. Two principal genetic pathways underlie the development of CRC. Most CRC tumors exhibit chromosomal instability associated with the loss of heterozygosity at multiple tumor suppressor loci such as $5 q, 17 p$, and 18q [3]. The microsatellite instability (MSI) refers to the mutational signature found in CRCs that evolve as a result of the inactivation of the DNA mismatch repair (MMR) system and is observed in approximately $15 \%$ of all CRCs. Furthermore, approximately 3\% of all CRCs arise as a consequence of Lynch syndrome, and nearly all Lynch syndrome CRCs exhibit MSI [4]. An- 
other $12 \%$ of CRCs represent the noninherited form of DNA MMR inactivation that is induced by methylation and, hence, silences the MLH1 gene promoter [5]. MSI-high (H) CRCs have a number of distinct features compared to MSI-low (MSI-L) and MSI-stable (MSS) CRCs, with the former being associated, on average, with a younger age at diagnosis and with tumors that more frequently have a mucinous phenotype, a poorly differentiated histology, and a proximal location and that show peritumoral lymphocytic infiltration [6].

Along with these distinguishing clinical characteristics, most previous studies reported that patients with MSI-H CRCs survive longer than do stage-matched patients with cancers exhibiting MSI-L/ S [6-11]. Furthermore, the MSI status has been reported to have predictive value for the effectiveness of adjuvant chemotherapy, as for MSI-H patients, adjuvant chemotherapy with 5-fluorouracil (5FU) has no benefit over surgery alone $[12,13]$. Therefore, in this study, we assessed the clinicopathologic features and prognoses of MSI-H CRC patients who underwent curative surgery.

\section{METHODS}

In this study, which was approved by the Institutional Review Board of the hospital and in which written informed consent for tissue collection was obtained from all patients, we analyzed a prospectively-collected series of 245 consecutive CRC patients who underwent a curative resection for pathologically-confirmed stages II or stage III CRCs at the Korea Cancer Center Hospital between 2006 and 2009. Patients were excluded if they had hereditary nonpolyposis CRC that met the Amsterdam II criteria, familial adenomatous polyposis, or a previous history of treatment for a malignancy. In order to analyze the MSI status, we amplified DNA extracted from formalin-fixed and paraffin-embedded tissue samples by using a polymerase chain reaction and a previously-recommended panel of markers (bat26, bat25, D5S346, D2S123, and D7S250) for genetic classification [14]. MSI-H was defined as instability in two or more markers, MSI-L as instability in a single marker, and MSS as no evidence of instability in the markers. The pathological staging of cancer was assessed postoperatively according to the seventh edition of the American Joint Committee on Cancer TNM grading system [15]. We described the location of a tumor as being in the proximal colon when it was located proximal to the splenic flexure.

Two 5-FU-based regimens were used for adjuvant chemotherapy. The first was the FL regimen, comprising 5-FU $\left(500 \mathrm{mg} / \mathrm{m}^{2}\right)$ and leucovorin $\left(20 \mathrm{mg} / \mathrm{m}^{2}\right)$ for 5 days every month for 6 cycles. The second was the FOLFOX4 regimen that consisted of 5-FU (400 $\mathrm{mg} / \mathrm{m}^{2}$ for loading, $600 \mathrm{mg} / \mathrm{m}^{2}$ for maintenance) and leucovorin $\left(200 \mathrm{mg} / \mathrm{m}^{2}\right)$, each for 2 days every 2 weeks. Oxaliplatin $(85 \mathrm{mg} /$ $\mathrm{m}^{2}$ ) was administered on the first day and repeated for 12 cycles. Patients who were administered more than $50 \%$ of the planned schedule of chemotherapy were entered into the adjuvant chemotherapy group. Thirty-seven patients did not receive chemotherapy because of their poor performance status or their refusal to undergo this treatment.

For detection of recurrence during the follow-up period, the carcinoembryonic antigen level and a computed tomography scan were checked every three months for the first year after the operation, and every six months thereafter. The median follow-up duration for all patients was 38 months.

Statistical analysis was performed using SPSS ver. 14.0 (SPSS Inc., Chicago, IL, USA). All results in the text and tables are given as mean \pm standard deviation. Categorical variables were analyzed using the chi-square test or Fisher exact test. Comparisons of quantitative variables between groups were made using the independent sample t-test. The Kaplan-Meier method was used for survival analysis, and the log rank test was used to determine any difference in survival between the groups. The Cox proportional hazard model with a backward elimination method was used for multivariate analyses. In all cases, a P-value of less than 0.05 was considered statistically significant.

\section{RESULTS}

Of the 245 patients included in this study, 20 (8.2\%) were found to have MSI-H tumors, and the remaining 225 patients had MSS or MSI-L tumors. Patients with MSI-H tumors were younger on average than were patients with MSI-L/S tumors, with a greater proportion being under 50 years of age, although this difference was not statistically significant. Furthermore, MSI-H primary tumors were located proximal to the splenic flexure significantly more frequently than MSI-L/S tumors ( $90.0 \%$ vs. $19.1 \%, \mathrm{P}<0.0001)$, and MSI-H CRC patients more frequently had a family history of colon or colon-related cancer in first-degree relatives $(25.0 \%$ vs. $8.4 \%, \mathrm{P}=$ $0.03)$. A further significant difference was in the lower frequency of lymph node metastasis in patients with MSI-H CRCs (55.6\% vs. 25.0\%, $\mathrm{P}=0.01$ ) (Table 1).

A pathological analysis revealed that the primary tumor size in the MSI-H group was $7.9 \pm 3.1 \mathrm{~cm}$, which was significantly larger than that in the MSI-L/S group $(5.2 \pm 2.0 \mathrm{~cm}, \mathrm{P}=0.001) . \mathrm{MSI}-\mathrm{H}$ CRCs showed an expansive growth pattern significantly more frequently (50.0\% vs. $28.6 \%, \mathrm{P}=0.05)$ than MSI-L/S tumors and more often had a poorly differentiated or a mucin-containing histology (35.0\% vs. $7.1 \%, P=0.001)$. The number of retrieved lymph nodes was also significantly higher for MSI-H CRCs than for MSIL/S CRCs ( $26.3 \pm 13.1$ vs. $20.7 \pm 11.2, \mathrm{P}=0.04)$, although the frequency of peritumoral lymphoid reaction was not significantly different between the groups (Table 2).

Table 3 summarizes the adjuvant chemotherapy administered to patients with MSI-H CRCs or MSI-L/S CRCs. Thirteen patients $(86.7 \%)$ received adjuvant chemotherapy for stage II disease, 11 (73.3\%) of whom received the FL regimen and 2 (13.3\%) received FOLFOX4. Adjuvant chemotherapy was administered to 88 of the patients (87.0\%) with MSI-L/S stage II CRC and to a similar proportion of patients with MSI-H stage II CRC. The distributions of 
Table 1. Clinical features of the entire study cohort

\begin{tabular}{lccc}
\hline Variable & MSI-H $(\mathrm{n}=20)$ & MSI-L/S $(\mathrm{n}=225)$ & P-value \\
\hline Age $(\mathrm{yr})$ & $59.1 \pm 16.0$ & $62.5 \pm 10.5$ & 0.35 \\
Age $<50 \mathrm{yr}$ & $6(30.0)$ & $31(13.8)$ & 0.09 \\
Male gender & $13(65.0)$ & $129(57.0)$ & 0.51 \\
Location & & & \\
$\quad$ Proximal & $18(90.0)$ & $43(19.1)$ & $<0.0001$ \\
$\quad$ Distal & $2(10.0)$ & $182(80.9)$ & \\
Synchronous CRC & $2(10.0)$ & $11(4.9)$ & 0.29 \\
Obstruction & $7(35.0)$ & $43(19.1)$ & 0.14 \\
Family history & $5(25.0)$ & $19(8.4)$ & 0.03 \\
$\quad$ first-degree relatives) & & & 0.19 \\
CEA (ng/mL) & $5.6 \pm 5.9$ & $11.2 \pm 20.2$ & 0.01 \\
Stage II & $15(75.0)$ & $100(44.4)$ & \\
Stage III & $5(25.0)$ & $125(55.6)$ & \\
\hline
\end{tabular}

Values are presented as mean \pm standard deviation or number (\%).

MSI-H, microsatellite instability-high; MSI-L/S, microsatellite instability-low/stable; $\mathrm{CRC}$, colorectal cancer; CEA, carcino-embryonic antigen.

Table 2. Pathological features of the entire study cohort

\begin{tabular}{lccc}
\hline Variable & MSI-H $(\mathrm{n}=20)$ & MSI-L/S $(\mathrm{n}=225)$ & P-value \\
\hline Tumor size (cm) & $7.9 \pm 3.1$ & $5.2 \pm 2.0$ & 0.001 \\
T4 & $3(15.0)$ & $20(8.9)$ & 0.41 \\
T4a & $2(10.0)$ & $17(7.6)$ & \\
T4b & $1(5.0)$ & $3(1.3)$ & \\
Growth pattern & & & \\
Expansive & $10(50.0)$ & $64(28.6)$ & 0.05 \\
$\quad$ Infiltrating & $10(50.0)$ & $160(71.4)$ & \\
Peritumoral lymphoid reaction & $3(15.0)$ & $33(14.7)$ & 1 \\
Histology & & & \\
$\quad$ WD/MD & $13(65.0)$ & $209(92.9)$ & 0.001 \\
PD/MUC & $7(35.0)$ & $16(7.1)$ & \\
Lymphatic invasion & $9(45.0)$ & $67(29.8)$ & 0.21 \\
Vascular invasion & $1(5.0)$ & $51(22.7)$ & 0.09 \\
Perineural invasion & $1(5.0)$ & $77(34.2)$ & 0.005 \\
No. of retrieved lymph nodes & $26.3 \pm 13.1$ & $20.7 \pm 11.2$ & 0.04 \\
\hline
\end{tabular}

Values are presented as mean \pm standard deviation or number (\%).

MSI-H, microsatellite instability-high; MSI-L/S, microsatellite instability-low/stable; WD/MD, well differntiation/moderate differentiation; PD/MUC, poor differentiation/ mucinous carcinoma.

the chemotherapy regimens were not significantly different. Of the 5 patients with MSI-H stage III CRC, 1 (20.0\%) was treated using the FL regimen, and $2(40.0 \%)$ received FOLFOX4 chemotherapy. In the MSI-L/S group, 104 of the patients (83.2\%) received
Table 3. Adjuvant chemotherapy after curative resection

\begin{tabular}{lcclll}
\hline & \multicolumn{2}{c}{ Stage $\|^{\mathrm{a}}$} & & \multicolumn{2}{c}{ Stage III } \\
\cline { 2 - 3 } \cline { 5 - 6 } & MSI-H & MSI-L/S & & MSI-H & MSI-L/S \\
\hline None & $2(13.3)$ & $12(12.0)$ & & $2(40.0)$ & $21(16.8)$ \\
FL & $11(73.3)$ & $79(79.0)$ & & $1(20.0)$ & $26(20.8)$ \\
FOLF0X4 & $2(13.3)$ & $9(9.0)$ & & $2(40.0)$ & $78(62.4)$ \\
\hline
\end{tabular}

Values are presented as number (\%).

MSI-H, microsatellite instability-high; MSI-L/S, microsatellite instability-low/stable; FL, 5-FU, leucovorin; FOLFOX4, 5-FU, leucovorin, oxaliplatin.

${ }^{\mathrm{a} P}=0.69 .{ }^{\mathrm{b} P}=0.27$.

Table 4. Location of recurrence

\begin{tabular}{lcccc}
\hline Location & MSI-H & MSI-L/S & Total & P-value \\
\hline Lung & $1(5.0)$ & $17(7.6)$ & $18(7.3)$ & 1 \\
Liver & $0(0)$ & $9(4)$ & $9(3.7)$ & 1 \\
Distant lymph nodes & $0(0)$ & $8(3.6)$ & $8(3.3)$ & 1 \\
Local recurrence & $1(5.0)$ & $6(2.7)$ & $7(2.9)$ & 0.45 \\
Peritoneum & $1(5.0)$ & $2(0.9)$ & $3(1.2)$ & 0.23 \\
Total & $3(15)$ & $39(17.3)$ & $42(17.1)$ & 1 \\
\hline
\end{tabular}

Values are presented as number (\%).

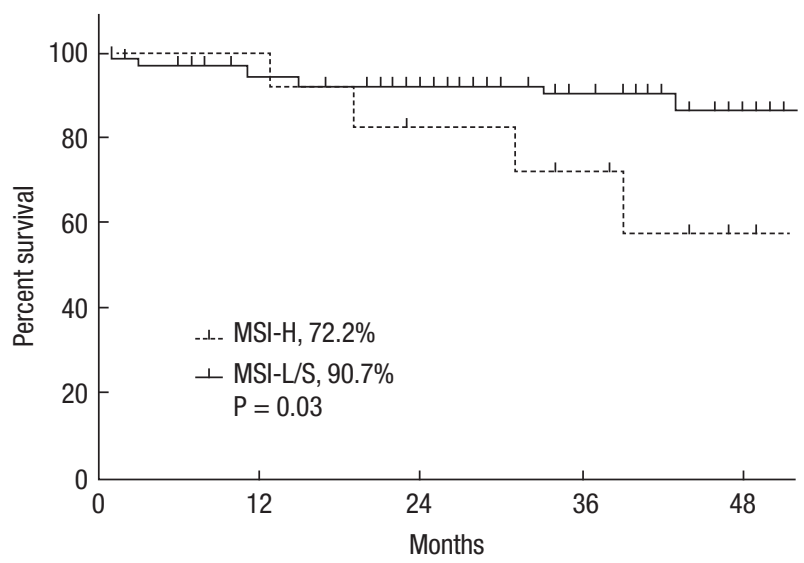

Fig. 1. Kaplan-Meier analysis of disease-free survival in the stage II subgroup. MSI-H, microsatellite instability-high; MSI-L/S, microsatellite instability-low/stable.

adjuvant chemotherapy, which was FOLFOX4 in 78 of the patients (62.4\%). A higher proportion of stage III MSI-L/S CRC patients underwent chemotherapy, although this difference was not statistically significant $(\mathrm{P}=0.27)$.

During the follow-up period, 42 patients $(17.1 \%)$ showed recurrence, most commonly in the lung $(\mathrm{n}=17,7.3 \%)$ and liver $(\mathrm{n}=9$, 3.7\%). However, the frequency and the site of recurrence did not differ significantly according to MSI status (Table 4 ).

A Kaplan-Meier analysis revealed that the 3-year disease-free survival (DFS) rates of the MSI-H and MSI-L/S groups were $77.5 \%$ and $79.6 \%$, respectively $(\mathrm{P}=0.78)$ and that the 3 -year overall sur- 


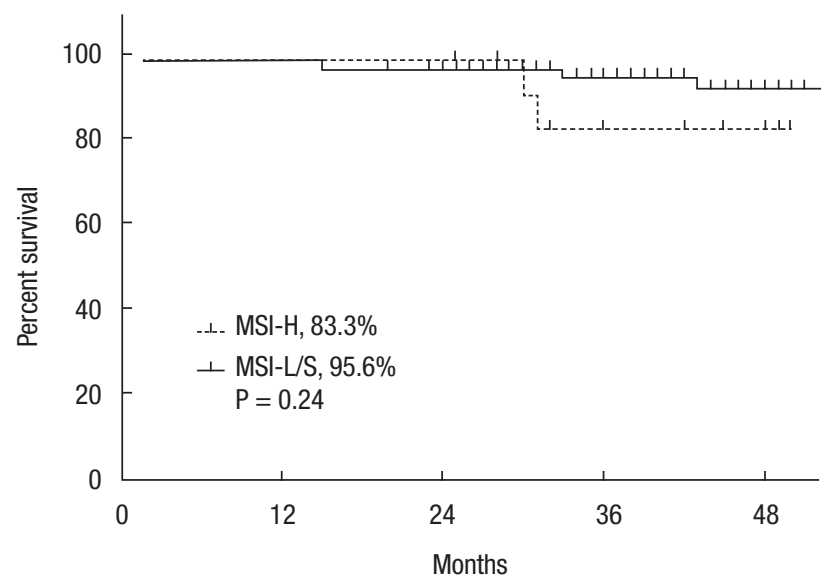

Fig. 2. Kaplan-Meier analysis of overall survival in the stage II subgroup. MSI-H, microsatellite instability-high; MSI-L/S, microsatellite instability-low/stable.

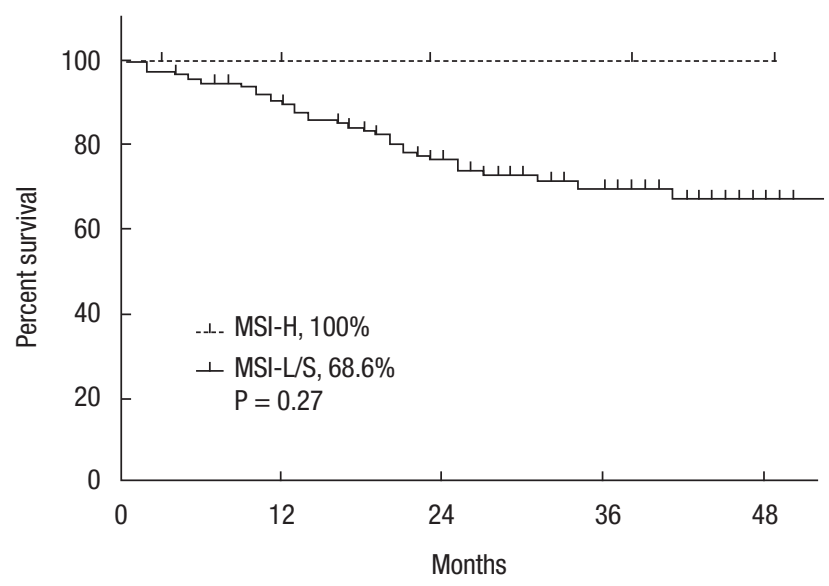

Fig. 3. Kaplan-Meier analysis of disease-free survival in the stage III subgroup. MSI-H, microsatellite instability-high; MSI-L/S, microsatellite instability-low/stable.

vival (OS) rates of the 2 groups were similar ( $87.5 \%$ vs. $92.0 \%$; $\mathrm{P}=$ 0.95). However, amongst patients with stage II disease, the DFS of the MSI-H group was $72.2 \%$, which was significantly worse than that of the MSI-L/S group (90.7\%, P = 0.03) (Fig. 1). The OS of the MSI-H group was also worse, but this difference was not statistically significant ( $83.3 \%$ vs. $95.6 \%, \mathrm{P}=0.24)$ (Fig. 2 ). In the stage III subgroup, the OS and the DFS were longer in the MSI-H group than in the MSI-L/S group, although these differences were not statistically significant (Figs. 3 and 4). A multivariate Cox analysis of the DFS in the stage II subgroup, in which the confounding effect of other risk factors was accounted for, revealed that MSI-H was an independent factor for a poor prognosis (adjusted hazard ratio $[\mathrm{aHR}], 4.0 ; 95 \%$ confidence interval $[\mathrm{CI}], 1.0-15.6, \mathrm{P}=$ 0.046 ), as was lymphatic invasion (aHR, 3.7; 95\% CI, 1.16-11.88; $\mathrm{P}=0.027$ ) (Table 5).

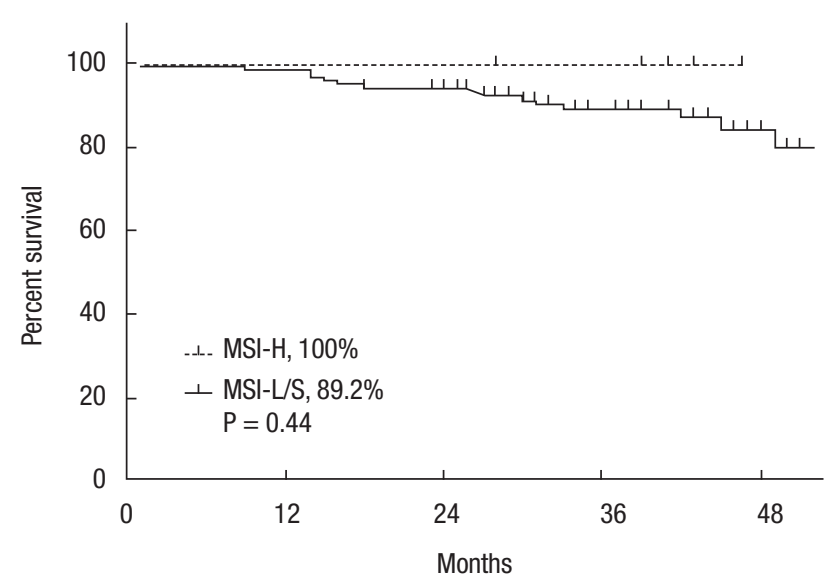

Fig. 4. Kaplan-Meier analysis of overall survival in the stage III subgroup. MSI-H, microsatellite instability-high; MSI-L/S, microsatellite instability-low/stable.

\section{DISCUSSION}

Following the initial discovery of MSI in patients with Lynch syndrome and in approximately $15 \%$ of patients with sporadic CRC tumors, considerable data have been accumulated and have demonstrated an improved outcome for patients with MMR-deficient CRC compared to those with MMR-proficient tumors or tumors with chromosomal instability. Patients with MSI-H tumors have distinct clinical and pathological features, irrespective of their germ line or sporadic origins [16]. These features include proximal colon predominance, frequent poor differentiation, mucinous histology, and an increased number of tumor-infiltrating lymphocytes [6]. In agreement with previous studies, we found that MSI-H CRC cases had different clinical and pathological characteristics compared to MSI-L/S CRC cases. In the former, the tumors were more frequently located in the proximal colon, were larger on average, had an expansive growth pattern more frequently, and were associated with a higher number of retrieved lymph nodes and less frequent lymph node metastasis. MSI-H CRCs were also frequently associated with mucinous histology and poor differentiation.

However, an important finding in our study was that patients with stage II disease and MSI-H tumors had poorer survival outcomes than those with an MSI-S tumor. Most studies, including a recent one on a large consecutive Norwegian series, showed better survival for MSI-H CRCs [17]. However, some reports showed a different result after 5-FU-based adjuvant chemotherapy in patients with MSI-H CRCs $[12,13]$. Ribic et al. [12] published a report describing 570 patients with colon cancer enrolled in 5 prior phase III trials of 5-FU-based adjuvant chemotherapy following curative resection for stage II and stage III colon cancer. In their analysis, MSI-H patients not treated with chemotherapy showed significantly improved survival. However, in the group of patients 
Table 5. Cox proportional hazard analysis of DFS in the stage II subgroup by using univariate and multivariate analyses

\begin{tabular}{|c|c|c|c|c|c|c|}
\hline \multirow{2}{*}{ Variable } & \multicolumn{3}{|c|}{ Univariate } & \multicolumn{3}{|c|}{ Multivariate } \\
\hline & HR & $95 \% \mathrm{Cl}$ & P-value & $\mathrm{HR}$ & $95 \% \mathrm{Cl}$ & P-value \\
\hline Age $\geq 65$ yr & 0.71 & $0.23-2.18$ & 0.551 & 0.62 & $0.19-2.08$ & 0.442 \\
\hline Male gender & 0.88 & $0.27-2.86$ & 0.826 & 0.88 & $0.27-2.92$ & 0.836 \\
\hline MSI-H & 3.36 & $1.04-10.93$ & 0.044 & 4.00 & $1.03-15.64$ & 0.046 \\
\hline Obstruction & 2.03 & $0.56-7.42$ & 0.283 & 1.64 & $0.42-6.40$ & 0.479 \\
\hline Lymphatic invasion & 5.00 & $1.68-14.89$ & 0.004 & 3.72 & $1.16-11.88$ & 0.027 \\
\hline Retrieved lymph nodes $<12$ & 2.22 & $0.72-6.79$ & 0.164 & 2.95 & $0.80-10.81$ & 0.103 \\
\hline $\mathrm{CEA}>7 \mathrm{ng} / \mathrm{mL}$ & 2.10 & $0.70-6.24$ & 0.184 & 3.07 & $0.90-10.43$ & 0.072 \\
\hline Adjuvant chemotherapy & 1.17 & $0.15-9.06$ & 0.878 & & & \\
\hline Vascular invasion & 1.67 & $0.37-7.59$ & 0.507 & & & \\
\hline Perineural invasion & 1.31 & $0.40-4.25$ & 0.656 & & & \\
\hline PD MUC & 0.71 & $0.09-5.47$ & 0.743 & & & \\
\hline T4 & 0.90 & $0.12-6.96$ & 0.922 & & & \\
\hline
\end{tabular}

DFS, disease-free survival; HR, hazard ratio; Cl, confidence interval; MSI-H, microsatellite instability-high; CEA, carcino-embryonic antigen; PD, poor differentiation; MUC, mucinous carcinoma.

who received adjuvant chemotherapy, MSI-H was not associated with an improved OS (hazard ration [HR], 1.07; 95\% CI, 0.62$1.86 ; \mathrm{P}=0.8$ ), although the benefit of treatment did differ significantly according to the tumor MSI patients with non-MSI-H tumors (HR, 0.72; 95\% CI, 0.53-0.99; P = 0.04) [12]. Moreover, Sargent et al. [13] recently confirmed and extended the initial findings of Ribic et al. [12] with a large retrospective study including samples obtained from six randomized, controlled trials. Their study evaluated the benefit of 5-FU-based adjuvant chemotherapy in patients with stage II CRC, excluding regimens combining oxaliplatin, irinotecan, and oral fluoropyrimidines. Stage III patients were not included in that analysis as adjuvant chemotherapy was already the standard of care. The authors concluded that $5 \mathrm{FU}$ did not benefit the patients and that, indeed, it might even be harmful for stage II CRC patients with MSI tumors. In the present study, we performed adjuvant chemotherapy to treat stage II CRC (87.8\%) as often as we did to treat stage III CRC (82.3\%). Considering that the majority of stage II patients were administrated the adjuvant FL regimen, the poor outcome for stage II MSI-H CRCs might be attributable to a relationship between the efficacy of adjuvant 5-FU-based chemotherapy and MSI status. Therefore, we assume that the nonbeneficial effect of 5-FU for MSI-H stage II patients and the improved survival of MSI-L/S patients who were administered the same adjuvant chemotherapy might make a significant contribution to a worse DFS. A multivariate analysis of the stage II subgroup revealed that MSI-H, after adjusting for other high risk factors, was an independent factor for a poor prognosis. A recent study about the use of molecular biomarkers for making adjuvant therapy decisions in stage II CRCs showed results in accordance with our findings. According to their report, while the MMR status had no influence on cancer progression in the surgery-alone group, defective MMR, along with CpG island methylation phenotype status, was the most important predictor of poor survival in the Cox regression analysis of the 5-FU treatment group [18].

The issue about adjuvant chemotherapy to treat stage II CRC has been controversial. Nevertheless, we have recommended adjuvant FL chemotherapy to treat stage II colon cancer for the lowrisk group and both FOLFOX and FL regimens for high-risk patients for following reasons: In the MOSAIC trial, which evaluated the benefit of the adjuvant FOLFOX over the FL regimen, recommended FOLFOX for high-risk stage II patients [19]. The characteristics of high-risk patients are T4 tumors; tumors with a low histological grade (for example, poorly differentiated and undifferentiated carcinomas); lymphatic, vascular or neural invasion; obstruction or perforation; and less than 10 harvested lymph nodes. Also, the QUASAR study did show a significant benefit of using adjuvant chemotherapy with 5-FU for stage II colon cancer [20], and our previous report on adjuvant chemotherapy to treat stage II colon cancer showed that stage II patients who received adjuvant chemotherapy with the FL regimen had significantly improved survival over the nonchemotherapy patients in both the low and the high risk of recurrence groups [21].

Several biological mechanisms have been proposed to explain why 5 -FU-based adjuvant chemotherapy fails to benefit patients with a defective MMR system. These include an antitumor immune response involving the lymphocytic infiltration characteristic of MSI-H tumors [22], which may be diminished by the immunosuppressive effects of chemotherapy. In vitro studies have also predicted a differential efficacy of 5-FU with respect to MMR 
status. Those studies found a recovery of chemoresistance to 5 -FU for hypermethylated defective MMR CRC cell lines that had been treated with demethylating agents $[23,24]$. A second possibility relates to the role of MMR systems in the removal of 5-FU from DNA, whereby the absence of these systems may reduce DNA repair and, thus, attenuate the effect of 5-FU [25]. It has been suggested that adding oxaliplatin to this regimen may overcome the negative effect of 5-FU on the DFS of patients with MSI-H CRCs [26]. In our study, despite more MSI-L/S patients with stage III disease receiving FOLFOX4 chemotherapy, there was no significant difference in survival. Although our findings need to be interpreted with caution because of the small number of patients, survival was better in the MSI-H patients. Whether the lack of benefit from adjuvant 5-FU in patients with MMR-defective tumors translates into a lack of benefit from FOLFOX has not been conclusively established. However, several studies have reported that MMR deficiency is not prognostic of the outcome in CRC patients who have received FOLFOX and even showed an improved outcome when using FOLFOX versus 5-FU alone [26, 27].

The major limitation of our study is the small number of enrolled MSI-H CRC cases, which made subgroup analysis difficult. Only $16 \mathrm{MSI}-\mathrm{H}$ patients received adjuvant chemotherapy; thus, we did not have enough cases to subgroup the patients according to stage, treatment with adjuvant chemotherapy, kind of chemo-regimen, and other clinico-pathological risk factors for recurrence. Therefore, the predictive value of MSI-H tumors for adjuvant chemotherapy could not be evaluated with satisfactory statistical power in either the univariate or the multivariate analysis. Indeed, the frequency of MSI-H CRC in Korea has been lower than the frequencies previously reported by Western countries [28]. According to a review of this topic from Western countries, evaluating the effect of adjuvant chemotherapy on MSI-H cancers is difficult because of the low number of sporadic MSI-H CRC, resulting in a limited statistical significance for MSI- $\mathrm{H}$ patients. The conflicting evidence on the benefit of 5-FU chemotherapy for treating MSI-H CRC patients relies mainly on a retrospective case series in which selection bias was a potential confounder [29]. Therefore, in Korea, practically, revealing the predictive role of MSI-H CRCs in adjuvant chemotherapy will be difficult based on only our single-center experience.

In summary, we identified a number of clinical and pathological features that differed significantly between CRC patients with and without MSI-H tumors. Also, MSI-H CRC showed worse survival results and an independent poor prognostic factor in stage II patients. We assume that these results might be due to 5-FU therapy having a different effect on MSI-H CRCs. However, this single-institutional retrospective study with a limited number of cases cannot be used to conclude that 5-FU-based adjuvant chemotherapy is actually of no benefit for patients with MSI-H CRCs. We believe that a large-scale well-designed multicenter study would be able to identify those features of MSI-H CRCs that can act as prognostic and predictive markers.

\section{CONFLICT OF INTEREST}

No potential conflict of interest relevant to this article was reported.

\section{REFERENCES}

1. Jung KW, Park S, Won YJ, Kong HJ, Lee JY, Park EC, et al. Prediction of cancer incidence and mortality in Korea, 2011. Cancer Res Treat 2011;43:12-8.

2. Fielding LP, Pettigrew N. College of American Pathologists Conference XXVI on clinical relevance of prognostic markers in solid tumors. Report of the Colorectal Cancer Working Group. Arch Pathol Lab Med 1995;119:1115-21.

3. Aaltonen LA, Peltomaki P, Leach FS, Sistonen P, Pylkkanen L, Mecklin JP, et al. Clues to the pathogenesis of familial colorectal cancer. Science 1993;260:812-6.

4. Boland CR. Evolution of the nomenclature for the hereditary colorectal cancer syndromes. Fam Cancer 2005;4:211-8.

5. Kane MF, Loda M, Gaida GM, Lipman J, Mishra R, Goldman H, et al. Methylation of the hMLH1 promoter correlates with lack of expression of hMLH1 in sporadic colon tumors and mismatch repair-defective human tumor cell lines. Cancer Res 1997;57:808-11.

6. Boland CR, Goel A. Microsatellite instability in colorectal cancer. Gastroenterology 2010;138:2073-87.e3.

7. Jenkins MA, Hayashi S, O'Shea AM, Burgart LJ, Smyrk TC, Shimizu D, et al. Pathology features in Bethesda guidelines predict colorectal cancer microsatellite instability: a population-based study. Gastroenterology 2007;133:48-56.

8. Gryfe R, Kim H, Hsieh ET, Aronson MD, Holowaty EJ, Bull SB, et al. Tumor microsatellite instability and clinical outcome in young patients with colorectal cancer. N Engl J Med 2000;342:69-77.

9. Popat S, Hubner R, Houlston RS. Systematic review of microsatellite instability and colorectal cancer prognosis. J Clin Oncol 2005; 23:609-18.

10. Vilar E, Gruber SB. Microsatellite instability in colorectal cancerthe stable evidence. Nat Rev Clin Oncol 2010;7:153-62.

11. Watanabe T, Wu TT, Catalano PJ, Ueki T, Satriano R, Haller DG, et al. Molecular predictors of survival after adjuvant chemotherapy for colon cancer. N Engl J Med 2001;344:1196-206.

12. Ribic CM, Sargent DJ, Moore MJ, Thibodeau SN, French AJ, Goldberg RM, et al. Tumor microsatellite-instability status as a predictor of benefit from fluorouracil-based adjuvant chemotherapy for colon cancer. N Engl J Med 2003;349:247-57.

13. Sargent DJ, Marsoni S, Monges G, Thibodeau SN, Labianca R, Hamilton SR, et al. Defective mismatch repair as a predictive marker for lack of efficacy of fluorouracil-based adjuvant therapy in colon cancer. J Clin Oncol 2010;28:3219-26.

14. Boland CR, Thibodeau SN, Hamilton SR, Sidransky D, Eshleman JR, Burt RW, et al. A National Cancer Institute Workshop on Microsatellite Instability for cancer detection and familial predisposition: development of international criteria for the determination 
of microsatellite instability in colorectal cancer. Cancer Res 1998; 58:5248-57.

15. American Joint Committee on Cancer. Colon and rectum. In: Edge SB, Byrd DR, Compton CC, Fritz AG, Greene FL, Trotti A, editors. AJCC cancer staging manual. 7th ed. New York: Springer; 2009. p. 143-64.

16. Sinicrope FA. DNA mismatch repair and adjuvant chemotherapy in sporadic colon cancer. Nat Rev Clin Oncol 2010;7:174-7.

17. Merok MA, Ahlquist T, Royrvik EC, Tufteland KF, Hektoen M, $\mathrm{Sjo} \mathrm{OH}$, et al. Microsatellite instability has a positive prognostic impact on stage II colorectal cancer after complete resection: results from a large, consecutive Norwegian series. Ann Oncol 2013; 24:1274-82.

18. Donada M, Bonin S, Barbazza R, Pettirosso D, Stanta G. Management of stage II colon cancer: the use of molecular biomarkers for adjuvant therapy decision. BMC Gastroenterol 2013;13:36.

19. Andre T, Boni C, Navarro M, Tabernero J, Hickish T, Topham C, et al. Improved overall survival with oxaliplatin, fluorouracil, and leucovorin as adjuvant treatment in stage II or III colon cancer in the MOSAIC trial. J Clin Oncol 2009;27:3109-16.

20. Quasar Collaborative Group, Gray R, Barnwell J, McConkey C, Hills RK, Williams NS, et al. Adjuvant chemotherapy versus observation in patients with colorectal cancer: a randomised study. Lancet 2007;370:2020-9.

21. Jee SH, Moon SM, Shin US, Yang HM, Hwang DY. Effectiveness of adjuvant chemotherapy with 5-FU/leucovorin and prognosis in stage II colon cancer. J Korean Soc Coloproctol 2011;27:322-8.
22. Morris M, Platell C, Iacopetta B. Tumor-infiltrating lymphocytes and perforation in colon cancer predict positive response to 5-fluorouracil chemotherapy. Clin Cancer Res 2008;14:1413-7.

23. Arnold CN, Goel A, Boland CR. Role of hMLH1 promoter hypermethylation in drug resistance to 5-fluorouracil in colorectal cancer cell lines. Int J Cancer 2003;106:66-73.

24. Carethers JM, Chauhan DP, Fink D, Nebel S, Bresalier RS, Howell $\mathrm{SB}$, et al. Mismatch repair proficiency and in vitro response to 5-fluorouracil. Gastroenterology 1999;117:123-31.

25. Fischer F, Baerenfaller K, Jiricny J. 5-Fluorouracil is efficiently removed from DNA by the base excision and mismatch repair systems. Gastroenterology 2007;133:1858-68.

26. Kim ST, Lee J, Park SH, Park JO, Lim HY, Kang WK, et al. Clinical impact of microsatellite instability in colon cancer following adjuvant FOLFOX therapy. Cancer Chemother Pharmacol 2010;66:659-67.

27. Zaanan A, Cuilliere-Dartigues P, Guilloux A, Parc Y, Louvet C, de Gramont A, et al. Impact of p53 expression and microsatellite instability on stage III colon cancer disease-free survival in patients treated by 5 -fluorouracil and leucovorin with or without oxaliplatin. Ann Oncol 2010;21:772-80.

28. Oh JR, Kim DW, Lee HS, Lee HE, Lee SM, Jang JH, et al. Microsatellite instability testing in Korean patients with colorectal cancer. Fam Cancer 2012;11:459-66.

29. Sinicrope FA, Sargent DJ. Clinical implications of microsatellite instability in sporadic colon cancers. Curr Opin Oncol 2009;21: 369-73. 